\title{
Coupling of Small Lattice Polarons to Magnetic Field in Magnetoresistive Manganites
}

\author{
C. Meneghini (a, b), C. Castellano (a), Ashwani Kumar (c), Sugata Ray (c), \\ D. D. SARMa (c), and S. MobiLIo $\left.{ }^{1}\right)(b, d)$
}

(a) INFM clo ESRF, GILDA CRG, B.P. 220, F-38043 Grenoble, France

(b) Laboratori Nazionali di Frascati, INFN, P.O. Box 13, I-00044 Frascati, Italy

(c) Solid State and Structural Chemistry Unit, Indian Institute of Science, Bangalore 560012, India

(d) Dipartimento di Fisica Universita' di Roma, Tre via della Vasca Navale 84, I-00186 Roma

\begin{abstract}
We report Extended X-Ray Absorption Fine Structure measurements at the Mn K absorption edge on hole doped manganese oxides $\mathrm{La}_{1-x} \mathrm{Ca}_{x} \mathrm{MnO}_{3}$ in a wide temperature range above and below the metal-insulator transition temperature in presence of a magnetic field. In accordance with previous studies an anomalous increase in the Debye-Waller factors is detected at the phase transition in the first coordination shell made up of $\mathrm{O}$ atoms. This is due to a local structural deformation around $\mathrm{Mn}^{3+}$ atoms associated with the Jahn-Teller effect and the formation of polarons. The application of a magnetic field results in a variation of this deformation with a thermal behaviour that closely resembles that of the magnetoresistance. This confirms the relevance of the polarons on the conductivity properties of such materials. The magnetic field has a huge effect on the second coordination shell probably related with the change of the $\mathrm{Mn}-\mathrm{O}-\mathrm{Mn}$ angle upon application of the magnetic field.
\end{abstract}

\section{Introduction}

The perovskite manganese oxides $\mathrm{La}_{1-x} \mathrm{Ca}_{x} \mathrm{MnO}_{3}$ in the doping range $0.20<x<0.50$ undergo upon cooling a phase transition from a paramagnetic insulating phase to a ferromagnetic metallic one, exhibiting the so called colossal magnetoresistance effect, i.e. a huge decrease of the electrical resistance in an applied magnetic field [1]. The interconnection between magnetic and electronic transport properties has been interpreted in terms of the double exchange mechanism [2 to 4]. However the double exchange mechanism alone is not sufficient to explain not only the colossal magnetoresistance but also most of the other peculiar electronic, magnetic and structural properties observed in the phase diagram of these materials; therefore in recent theories also a strong coupling between conduction electrons and lattice is assumed [5 to 8]. In fact, the undoped compound $\mathrm{LaMnO}_{3}$ exhibits a Jahn-Teller lattice distortion around the $\mathrm{Mn}^{3+}$ ions, which is absent in the other extreme compound $\mathrm{CaMnO}_{3}$ where only $\mathrm{Mn}^{4+}$ ions are present. In the hole doped compounds, which have both $\mathrm{Mn}^{3+}$ and $\mathrm{Mn}^{4+}$ sites,

1) Corresponding author: Tel.: 0039-0694032288; Fax: 0039-0694032304; e-mail: Mobilio@Lnf.Infn.It 
the conduction electrons move from $\mathrm{Mn}^{3+}$ sites to $\mathrm{Mn}^{4+}$ ones carrying with them the Jahn-Teller distortion so being strongly coupled with the lattice. In spite of the extensive experimental and theoretical investigations performed on such systems, no clear conclusion has been reached up to now on the size and nature of this polaronic interaction, and various suggestions have been made ranging from small polarons (with sizes in the range of atomic spacings) to considerably larger polarons (spread out over several Mn sites). Moreover, it has also been suggested [7] that some physical properties of such materials in the insulating regime are not explained by lattice polarons, but by spin polarons. A magnetic nature of the polarons is confirmed by other studies as the observation of large and unexpected shifts in the magnetic properties with isotopic change of the oxygen ions which point to an interplay between the phonons and the spin of the system [9] or the small angle neutron scattering and thermal expansion studies [10] that have shown the presence of magnetic polarons above $T_{\mathrm{C}}$ with a size of about $12 \AA$, i.e. six times the interatomic $\mathrm{Mn}-\mathrm{O}$ spacings in these manganites. However other observations with neutron scattering [11] and Raman scattering [12] have not revealed the spin nature of the polarons. Thus, it appears that different experimental probes reach different conclusions on the nature and size of the polarons.

X-ray absorption spectroscopy particularly in the EXAFS regime is an ideal tool to probe the polarons directly. In fact, previous EXAFS experiments [13, 14] pointed out unambigously the rise of lattice distortions at the phase transition temperature, with the formation of polarons which remain alive well inside the paramagnetic phase.

To study in a direct way the coupling of the polarons to the magnetic field, we report in this paper an EXAFS study on Ca doped $\mathrm{La}_{1-x} \mathrm{Ca}_{x} \mathrm{MnO}_{3}$ manganites at the $\mathrm{Mn} \mathrm{K}$ edge in the presence of magnetic field.

\section{Experimental}

Four samples of $\mathrm{La}_{1-x} \mathrm{Ca}_{x} \mathrm{MnO}_{3}, x=0.1,0.25,0.33$ and 0.4 were prepared by conventional nitrate method using $\mathrm{La}_{2} \mathrm{O}_{3}, \mathrm{CaCO}_{3}$ and $\mathrm{MnC}_{2} \mathrm{O}_{4} \cdot 2 \mathrm{H}_{2} \mathrm{O}$. All the samples were characterized by X-ray diffraction, ac susceptibility, resistivity and magnetoresistance measurements.

The temperature dependent resistivity, measured using standard four probe dc technique, is shown in Fig. 1 for some samples. The $x=0.1$ sample appears to be an insulator $(\mathrm{d} R / \mathrm{d} T<0)$ in the whole temperature range while all other samples show the presence of a metal-insulator transition with evident resistivity maxima. For these samples the negative magnetoresistance, defined as $\mathrm{MR}=\left(R_{H=0}-R_{H}\right) / R_{H}$, measured with an applied magnetic field of $0.9 \mathrm{~T}$ presents a sharp peak at about the metal-insulator transition temperature.

Sample powders were milled and suspended in alcohol and then deposited on millipore membranes, to obtain very homogeneous samples. Mn K edge EXAFS data in the energy range 6.2 to $7.3 \mathrm{keV}$ were recorded in transmission geometry in the temperature range 77 to $360 \mathrm{~K}$ at the Italian CRG-GILDA beam line at ESRF (Grenoble). Beam energies were defined using a $\mathrm{Si}[311]$ double crystal monochromator working in dynamical sagittal focussing mode [15]. A Pd coated grazing incidence mirror system completely removed the harmonics from the incident beam. Permanent magnets were used to apply a magnetic field of $1.1 \mathrm{~T}$ to the sample; the field homogeneity was better than $0.5 \%$ at the sample stage. 


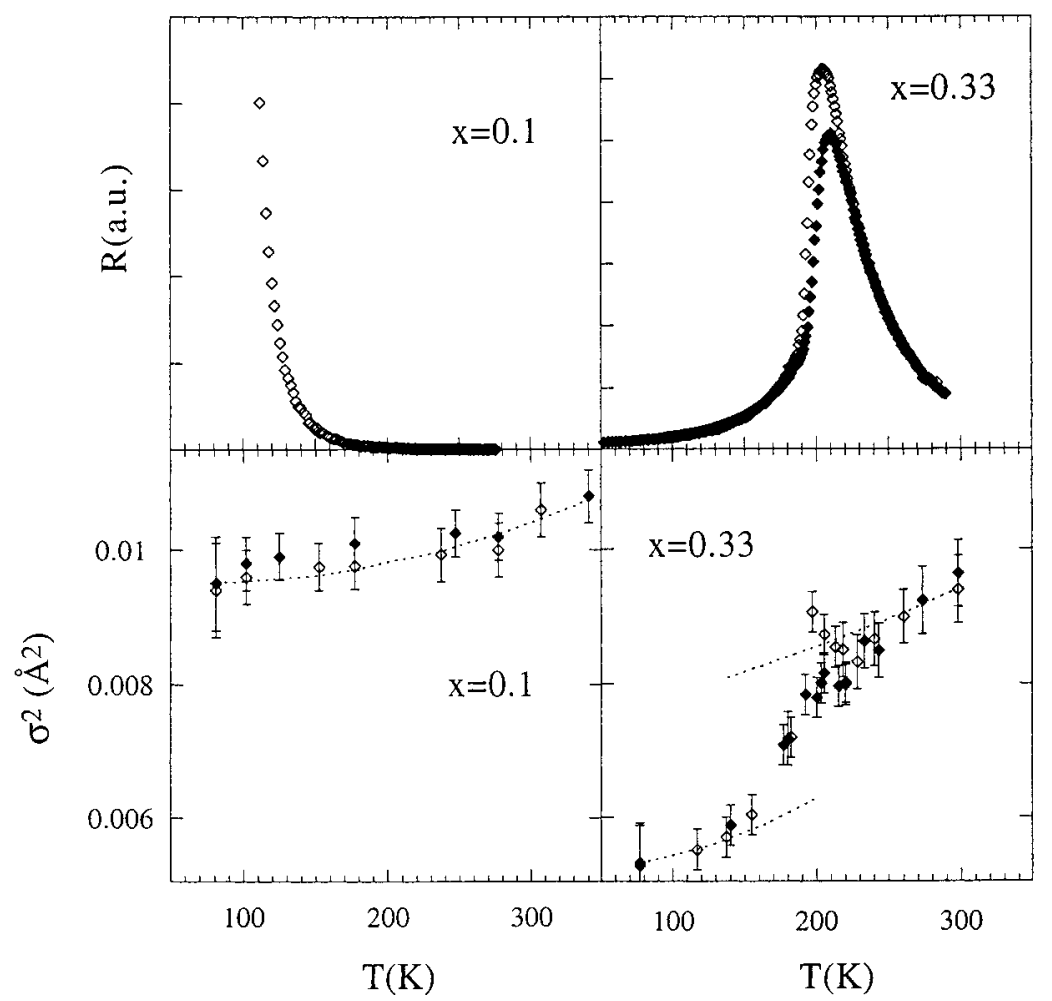

Fig. 1. Temperature dependence of resistance (upper panels) and Debye-Waller factors (lower panels) of $\mathrm{La}_{1-x} \mathrm{Ca}_{x} \mathrm{MnO}_{3}$ for samples $x=0.1$ and $x=0.33$, with (full symbols) and without (empty symbols) the magnetic field. The dotted lines on the Debye-Waller factors are fits of the experimental behaviour with a static contribution plus a phonon contribution

\section{Data Analysis and Results}

The EXAFS oscillations, $\chi(k)$, were extracted from the experimental data using standard procedures [16] and were normalized using Lengeler-Eisenberger method [17]. The $\chi(k)$ data were Fourier transformed with $k^{3}$ weight in the $\mathrm{k}$ range of 2.6 to $12 \AA^{-1}$. The Fourier transforms thus obtained (Fig. 2), uncorrected for the phase shift show many correlation peaks up to $R=8 \AA$, as expected for a well ordered crystalline structure. The nearest neighbour peak, at about $1.5 \AA$, corresponds to $\mathrm{Mn}-\mathrm{O}$ neighbours; the second intense peak at about $3.2 \AA$ includes contributions from $\mathrm{Mn}-\mathrm{Mn}$ and $\mathrm{Mn}-\mathrm{La}$ pairs.

Fig. 2 shows qualitatively the effect of the applied magnetic field; the second peak of the FT in the ferromagnetic phase $\left(T<T_{\mathrm{C}}\right)$ does not depend on $H$, while for temperature around the metal-insulator transition it shows large changes upon application of the magnetic field. Variations are present also in the first neighbour coordination shell, but are not visible in the FT and are detected only after a careful quantitative analysis. For this purpose the Mn-O first shell EXAFS oscillations, $\chi_{\mathrm{MnO}}(k)$, were isolated by inverse Fourier filtering in the $R$ range from 1 to $2.3 \AA$. The so obtained $\chi_{\mathrm{MnO}}(k)$ were 


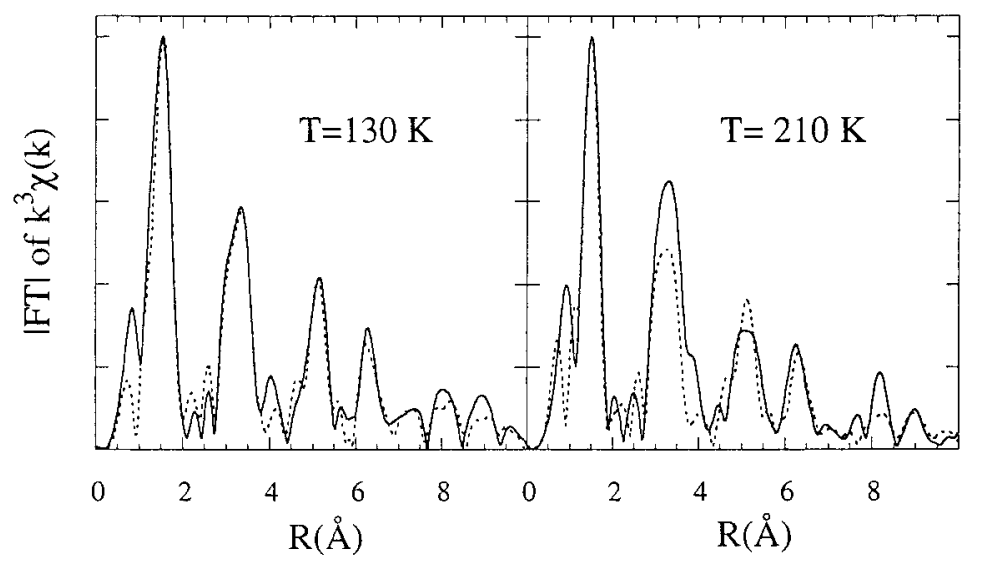

Fig. 2. Fourier transforms of the $k^{3}$ weighted EXAFS signal of the sample $\mathrm{La}_{0.67} \mathrm{Ca}_{0.33} \mathrm{MnO}_{3}$ obtained below $(T=130 \mathrm{~K})$ and around the Curie temperature $(T=210 \mathrm{~K})$ with a magnetic field of $0 \mathrm{~T}$ (full line) and $1.1 \mathrm{~T}$ (dotted line)

fitted to the standard EXAFS formula [16] using experimental amplitude and phase functions extracted from the data recorded at liquid nitrogen temperature. The use of experimental amplitude and phase functions simplifies the data analysis since a single shell model EXAFS function can be used although the $\mathrm{Mn}-\mathrm{O}$ bonds present a complex distribution, in which up to three coordination shells can be recognized [18]. In fitting the experimental data, the $\mathrm{Mn}-\mathrm{O}$ coordination number was fixed to 6 , so that the only variable parameters were $\mathrm{Mn}-\mathrm{O}$ bond lengths, $R_{\mathrm{Mn}-\mathrm{O}}$, and the mean square relative displacements of these bond lengths $\sigma_{\mathrm{Mn}-\mathrm{O}}^{2}=\left\langle\left(R_{\mathrm{Mn}-\mathrm{O}}^{\mathrm{i}}-R_{\mathrm{Mn}-\mathrm{O}}\right)^{2}\right\rangle$ where $R_{\mathrm{Mn}-\mathrm{O}}^{\mathrm{i}}$ is the bond length of a specific $\mathrm{Mn}-\mathrm{O}$ pair and the average is performed over all the pairs present in the sample. $R_{\mathrm{Mn}-\mathrm{O}}$ for each of the samples was found to be relatively independent of the temperature within the experimental accuracy. The values of $R_{\mathrm{Mn}-\mathrm{O}}$, averaged over all the temperatures for each sample, are found to decrease smoothly from $1.962 \pm 0.005$ to $1.950 \pm 0.005 \AA$ with increasing $x$ in agreement with earlier results [19], Moreover, $\left\langle R_{\mathrm{Mn}-\mathrm{O}}\right\rangle$ in each case is smaller by about $0.005 \AA$ in presence of the magnetic field compared to that with $H=0$, in agreement with the magnetostrictive effect observed in these materials [20]. In contrast to these expected behaviours of the $\mathrm{Mn}-\mathrm{O}$ bond lengths, the $\sigma_{\mathrm{Mn}-\mathrm{O}}^{2}$, probing the distortions in the $\mathrm{MnO}_{6}$ local octahedra, exhibit very unusual dependence on both temperature and magnetic field, as discussed below.

In Fig. 1, we report $\sigma_{\mathrm{Mn}-\mathrm{O}}^{2}$ of the two samples $x=0.1$ and $x=0.33$ as a function of temperature in absence of a magnetic field (open symbols) as well as in the presence of magnetic field (full symbols). This figure underlines the large difference between the samples exhibiting the metal-insulator transition $(x>0.20)$ and those that are insulating throughout the whole temperature range $(x<0.20)$. The $\sigma_{\mathrm{Mn}-\mathrm{O}}^{2}$ for the $x=0.1$ sample exhibits a slight monotonic decrease with temperature. This behaviour is very similar to the one reported for $\mathrm{LaMnO}_{3}$ [14] and can be described in terms of a correlated Debye model describing the temperature dependent part plus a temperature independent static disorder. The large static disorder (static component of $\sigma_{\mathrm{Mn}-\mathrm{O}}^{2}$ being about $0.009 \AA^{2}$ ) at this composition reflects the formation of polarons driven by the Jahn-Teller effect around $\mathrm{Mn}^{3+}$ sites for the insulating compositions. It is also evident that the 
application of a magnetic field has no effect on $\sigma_{\mathrm{Mn}-\mathrm{O}}^{2}$ suggesting the absence of any strong coupling of these Jahn-Teller polarons with magnetic field.

The values of $\sigma_{\mathrm{Mn}-\mathrm{O}}^{2}$ for the $x=0.25,0.33$ and 0.4 samples deviate from the expected Debye-like behaviour in the vicinity of $T_{\mathrm{C}}$ as already observed by other EXAFS works $[13,14]$. The $\sigma_{\mathrm{Mn}-\mathrm{O}}^{2}$ shows the expected decreasing trend (phonon-like) with decreasing temperature in the high and low temperature regions (dots): at low temperature it has rather small values $\left(\sigma_{\mathrm{Mn}-\mathrm{O}}^{2}=0.003\right.$ to $\left.0.005 \AA^{2}\right)$, typical of cubic systems (such as $\mathrm{MnO}$ ), showing that no distortion in the first coordination shell is present. On the other hand, large static distortions are found at high temperature for the presence of JahnTeller distorted Mn local environment. Near $T_{\mathrm{C}}$ the $\sigma_{\mathrm{Mn}-\mathrm{O}}^{2}$ presents a discontinuity due to the formation of polarons that mimicks the rapid change in the resistivity as discussed previously [14].

The effect of the applied magnetic field on the $\mathrm{Mn}-\mathrm{O}$ Debye-Waller factor is better seen in Fig. 3 where the variations of the disorder factors upon application of the magnetic field $\Delta \sigma_{H}^{2}$ are shown. They are obtained, for each temperature, by fitting the zero field data using the experimental amplitude and phase functions extracted from the $H=1.1 \mathrm{~T}$ data. In such a way an accurate evaluation of the relative changes of DebyeWaller factors is achieved. $\Delta \sigma_{H}^{2}$ show a well defined peak in a temperature range around the metal-insulator transition, indicating that the application of the field reduces the polaronic distortions around the manganese. As shown in the figure such peaks are strongly correlated to the magnetoresistance peaks, showing the existence of a strong correlation between the local distortion around $\mathrm{Mn}$, the conductivity and the magnetic properties: the magnetic field orders the magnetic moments so favouring the electron mobility and the reduction of the $\mathrm{Mn}-\mathrm{O}$ distortions.

The influence of the magnetic field on the local order is dramatically amplified when considering the neighbour coordination shell (Fig. 2). This is composed of eight La atoms at the center of the cubic cells of the perovskite structure and of six $\mathrm{Mn}$ at the corners. Nevertheless the interpretation of this peak is complicated by the presence of an almost collinear $\mathrm{Mn}-\mathrm{O}-\mathrm{Mn}$ configuration giving rise to a strong multiple scattering

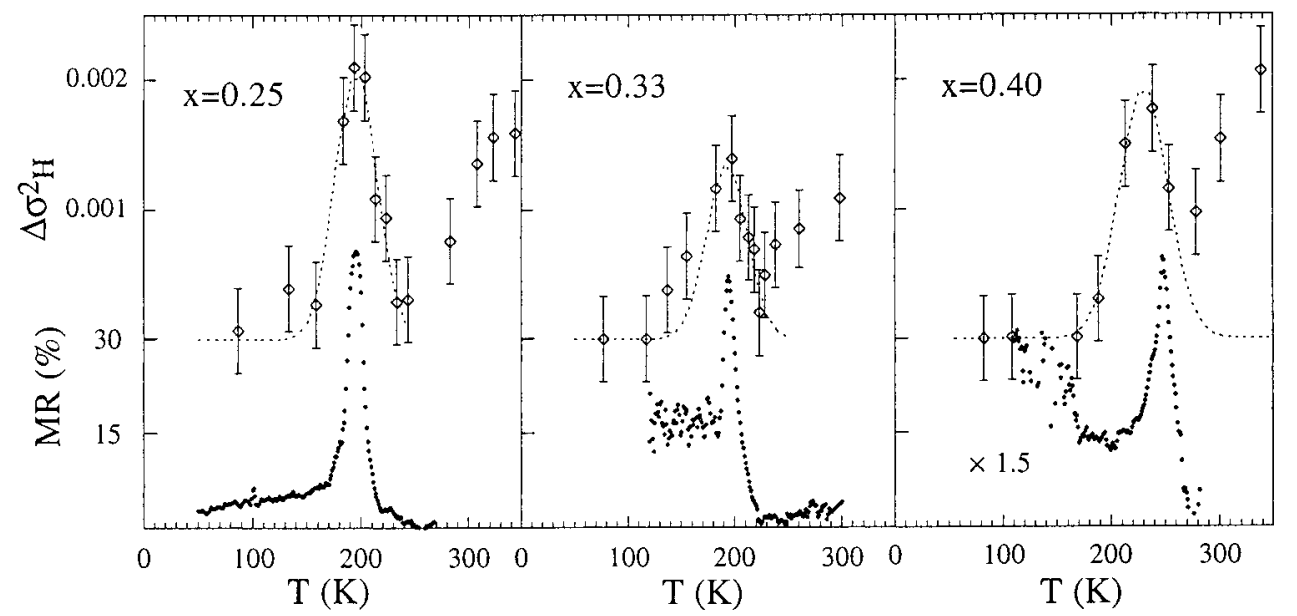

Fig. 3. The differences in the Debye-Waller factors (upper plots) obtained without and with the applied magnetic field (see text) compared with the magnetoresistance curves (lower plots) 
signal coming out from the so called "focussing effect" i.e. the strong scattering amplitude of the $\mathrm{O}$ atoms in the forward direction, that enhances the global contribution of the Mn next neighbour atoms; such effect is very sensitive to the $\mathrm{Mn}-\mathrm{O}-\mathrm{Mn}$ bonding angle. So the decrease of the second FT peak probably reflects the action of the applied magnetic field on the $\mathrm{Mn}-\mathrm{O}-\mathrm{Mn}$ bond angle: it is important to quantify such an effect since the electron hopping parameter is related to this angle [21]. A quantitative analysis of such peak within a multiple scattering approach is under way and will be reported in a forthcoming paper.

In conclusion we have probed the effects of the applied magnetic field on the local structure around $\mathrm{Mn}$ showing that the magnetic field reduces the $\mathrm{Mn}-\mathrm{O}$ bond distortions in the region of the metal to insulator transition and that changes occur in the $\mathrm{Mn}-\mathrm{O}-\mathrm{Mn}$ bond angle distribution; and that both these effects are strongly connected to the magnetoresistance properties.

\section{References}

[1] R. von Helmholt, J. Weker, B. Holzapfel, L. Schultz, and K. Samwer, Phys. Rev. Lett. 71, 2331 (1993).

[2] C. ZeNER, Phys. Rev. 81, 403 (1951).

[3] P. W. Anderson and H. Hasegawa, Phys. Rev. 100, 675 (1955).

[4] P. G. DE GENNES, Phys. Rev. 118, 141 (1960).

[5] A. J. Millis, B. Shraiman, and R. Mueller, Phys. Rev. Lett. 77, 175 (1996).

[6] H. Roder, J. Zhang, and A. Bishop, Phys. Rev. Lett. 76, 1356 (1996).

[7] C. M. VARMa, Phys. Rev. B 54, 7328 (1996).

[8] L. Sheng, D. Y. Xing, D. N. Sheng, and C. S. Ting, Phys. Rev. Lett. 76, 1356 (1997).

[9] G. Zhao, K. Conder, H. Keller, and K. A. Muller, Nature 381, 676 (1996).

[10] J. M. DE TERESA, et al., Nature 386, 256 (1997).

[11] S. J. L. Billinge, R. G. Di Francesco, G. H. Kwei, J. J. Neumeier, and J. D, Thompson, Phys. Rev. Lett. 77, 715 (1996).

[12] S. Yoon et al., Phys. Rev. B 58, 2795 (1998).

[13] C. H. Booth, F. Bridges, G. H. Kwei, J. M. Lawrence, A. L. Cornelius, and J. J. Neumeier, Phys. Rev. B 57, 10440 (1998).

[14] C. Meneghini, R. Cimino, S. Pascarelli, S. Mobilio, C. Raghu, and D. D. Sarma, Phys. Rev. B 56, 3520 (1997).

[15] S. Pascarelli, F. Boscherini, F. D’Acapito, J. Hrdy, C. Meneghini, and S. Mobilio, J. Synchrotron Radiation 33, 147 (1996).

[16] P. A. Lee, P. H. Citrin, P. Eisenberger, and B. M. Kincaid, Rev. Mod. Phys. 53, 769 (1981).

[17] B. Lengeler and P. Eisenberger, Phys. Rev. B 21, 4507 (1980).

[18] A. Lanzara, N. L. Saini, M. Brunelli, F. Natali, A. Bianconi, P. G. Radaelli, and S. W. Cheong, Phys. Rev. Lett. 81, 878 (1998).

[19] G. Subias, J. Garcia, J. Blasco, and M. G. Proietti, Phys. Rev. B 57, 748 (1998).

[20] M. R. Ibarra, P. A. Algarabel, C. Marquina, and J. Garcia, Phys. Rev. Lett. 75, 3541 (1995).

[21] P. G. Radaelli, G. Iannone, M. Marezio, H. Y. Hwang, S. W. Cheong, J. D. Jorgensen, and D. N. Argyriou, Phys. Rev. B 56, 8265 (1997). 